\title{
Bacterial identification by lipid profiling using liquid atmospheric pressure matrix- assisted laser desorption/ionization mass spectrometry
}

Article

Accepted Version

Lellman, S. E. and Cramer, R. (2019) Bacterial identification by lipid profiling using liquid atmospheric pressure matrixassisted laser desorption/ionization mass spectrometry. Clinical Chemistry and Laboratory Medicine, 58 (6). pp. 930938. ISSN 1437-4331 doi: https://doi.org/10.1515/cclm-20190908 Available at https://centaur.reading.ac.uk/88285/

It is advisable to refer to the publisher's version if you intend to cite from the work. See Guidance on citing.

To link to this article DOI: http://dx.doi.org/10.1515/cclm-2019-0908

Publisher: De Gruyter

All outputs in CentAUR are protected by Intellectual Property Rights law, including copyright law. Copyright and IPR is retained by the creators or other copyright holders. Terms and conditions for use of this material are defined in the End User Agreement. 


\section{CentAUR}

Central Archive at the University of Reading

Reading's research outputs online 


\section{Bacterial identification by lipid profiling using liquid atmospheric pressure}

matrix-assisted laser desorption/ionization mass spectrometry

Sophie E. Lellman ${ }^{1}$ and Rainer Cramer $^{1 *}$

${ }^{1}$ Department of Chemistry, University of Reading, Whiteknights, Reading, RG6 6AD, United Kingdom

*Address correspondence to:

Prof. Rainer Cramer, Department of Chemistry, University of Reading, Whiteknights, Reading RG6 6AD, UK.

Tel.: +44-118-378-4550; e-mail: r.k.cramer@reading.ac.uk

Keywords - Biotyping, lipids, bacteria, speciation, profiling, matrix-assisted laser desorption/ionization (MALDI), mass spectrometry 


\section{Abstract}

Background - In recent years, mass spectrometry (MS) has been applied to clinical microbial biotyping, exploiting the speed of matrix-assisted laser desorption/ionization (MALDI) in recording microbe-specific MS profiles. More recently, liquid atmospheric pressure (AP) MALDI has been shown to produce extremely stable ion flux from homogenous samples and 'ESI-like' multiply charged ions for larger biomolecules, whilst maintaining the benefits of traditional MALDI including high tolerance to contaminants, low analyte consumption and rapid analysis. These and other advantages of liquid AP-MALDI MS have been explored in this study to investigate its potential in microbial biotyping.

Methods - Genetically diverse bacterial strains were analyzed using liquid AP-MALDI MS, including clinically relevant species such as Escherichia coli, Staphylococcus aureus and Klebsiella pneumoniae. Bacterial cultures were subjected to a simple and fast extraction protocol using ethanol and formic acid. Extracts were spotted with a liquid support matrix (LSM) and analyzed using a Synapt G2-Si mass spectrometer with an in-house built APMALDI source.

Results - Each species produces a unique lipid profile in the $\mathrm{m} / \mathrm{z}$ range of $400-1100$, allowing species discrimination. Traditional (solid) MALDI MS produced spectra containing a high abundance of matrix-related clusters and an absence of lipid peaks. The MS profiles of the bacterial species tested form distinct clusters using principle component analysis (PCA) with a classification accuracy of $98.63 \%$ using a PCA-based prediction model.

Conclusions - Liquid AP-MALDI MS profiles can be sufficient to distinguish clinically relevant bacterial pathogens and other bacteria, based on their unique lipid profiles. The analysis of 
the lipid MS profiles is typically excluded from commercial instruments approved for clinical diagnostics. 


\section{Introduction}

Biotyping using mass spectrometry (MS) has proved highly beneficial in many sectors, including the food industry and clinical laboratories, overtaking traditional microbiological methods such as analysis by microscopy and biochemical assays. The crucial limiting factor of these classical methods is time, requiring after initial bacterial culture at least an additional 18 hours for a complete identification(1).

The use of MS for bacterial identification is now a vital tool in the clinical laboratory, leading to a vast reduction in identification turnaround time, from 24-48 hours to approximately one hour(2) following the growth period. Thus, the length of stay of patients in hospitals, as well as patient mortality, can be reduced, which in turn significantly reduces hospital costs per infection $(3,4)$ in comparison to traditional methods. Commercial MS instruments approved for clinical use utilize a (MALDI) source coupled to an axial time-of-flight (TOF) mass spectrometer. These instruments analyze the unique peptide/protein microbial fingerprint in the $\mathrm{m} / \mathrm{z}$ range of 2,000-12,000, which can provide species-level identification $(5,6)$. The range below $\mathrm{m} / \mathrm{z} 2,000$ is typically excluded from these scans due to the interference of matrix related ions, however this also excludes the detection of low molecular weight metabolites and lipids.

Lipids are essential macromolecules within the bacterial cell, being a major component of the phospholipid bilayer of the cell membrane, as well as having roles in storage and signalling pathways. The term 'lipidomics' was first coined by Han and Gross in 2003(7), encompassing the study of the cellular lipidome of biological samples, including bacterial cells. Most commonly, the analytical study of bacterial lipids is performed using pyrolysis MS (8-10), and more recently electrospray ionization (ESI) MS $(11,12)$. However there has also 
been research performed using MALDI-TOF MS (13). These and other MS techniques have demonstrated that certain strains can be distinguished based on their lipid profiles, lending the field of lipidomics to bacterial classification and identification $(14,15)$. However, only a few studies have explored the use of bacterial lipid profiles for clinical biotyping, and even less by using MALDI MS $(16,17)$.

Recent developments in liquid AP-MALDI have shown this technique to provide benefits beyond those of traditional MALDI, which is performed with solid samples under vacuum conditions. Liquid MALDI samples are typically comprised of a liquid support matrix (LSM), formed of matrix chromophore molecules and the addition of a viscous support liquid such as glycerol. Liquid MALDI samples have self-healing properties, which allow for a relatively stable ion flux with relatively low sample ablation (18). Liquid MALDI samples also have a greater homogenous distribution of matrix and analyte molecules than solid MALDI samples (19), enabling prolonged analyte ion detection and removing the need for the user to find a 'sweet-spot' on the sample for sufficient analyte ionization which is often needed with solid MALDI samples.

Another major advantage of the use of liquid AP-MALDI is the production of multiply charged ions, which is usually only obtained with an ESI source. This offers greater choice for $\mathrm{MS} / \mathrm{MS}$ analysis, including ETD/ECD, and provides low $\mathrm{m} / \mathrm{z}$ values for high-molecular weight biomolecules, thus facilitating the use of conventional, high-performing, hybrid ESI MS instrumentation. The mechanism behind the formation of multiply charged ions has been discussed elsewhere (20-22). However, despite having shown that with the addition of divalent metal cations liquid AP-MALDI can produce doubly charged lipids, diagnostically informative lipid profiles are easier obtained with singly charged lipids as shown in the 
analysis of biological samples such as milk extracts (23). The current main limitation of liquid AP-MALDI MS lies in the routine analysis of larger peptides and proteins above 30kDa from complex biological samples $(24,25)$.

This study demonstrates the novel application of liquid AP-MALDI for the profiling of bacterial lipids to provide species-level identification. We demonstrate that bacteria can be identified using their unique lipid mass fingerprint, providing a rapid analytical alternative for bacterial identification, which in clinical analysis is mostly performed using a peptidomic/proteomic mass fingerprint. The benefits of using liquid MALDI samples over solid MALDI samples to analyze bacterial extracts are demonstrated. Bacterial extracts were prepared using a simple ethanol/formic acid extraction protocol similar to clinical biotyping protocols for solid MALDI MS (26). Secondly, several bacterial species were analyzed to obtain a unique lipid profile for each bacterium to allow for species differentiation. These data were used to perform principle component analysis (PCA) to determine the differences between the MS profiles obtained for each bacterial species.

\section{Materials and Methods}

\subsection{Materials}

All MALDI matrix components and protein standards were purchased from Sigma-Aldrich (Gillingham, UK), besides porcine insulin which was purchased from VWR (Leighton Buzzard, UK). The peptide standard mixture was purchased from Bruker UK Ltd. (Coventry, UK) while the sodium iodide MS calibration solution was purchased from Waters (Wilmslow, UK). Water, formic acid and acetonitrile (all HPLC grade), as well as ethanol (reagent grade) were purchased from Fisher Scientific (Loughborough, UK). 


\subsection{Sample preparation}

First-generation bacterial strains (Pro-Lab Diagnostics, Merseyside, UK) were received in freeze-dried discs and revived according to recommended growth conditions from Public Health England which were obtained by searching for strain number (see Supplemental Material, Table S1) in the National Collection of Type Cultures (27). All culture media were obtained from Oxoid/ThermoFisher (Basingstoke, UK) and prepared according to manufacturer instructions, including autoclaving at $121^{\circ} \mathrm{C}$ for 15 minutes to ensure sterility. Following incubation at $37^{\circ} \mathrm{C}$ for 24 hours (or 72 hours in the case of L. brevis), bacterial growth was scraped off the surface of the media and resuspended in $300 \mu \mathrm{L}$ of HPLC grade water. A volume of $900 \mu \mathrm{L}$ of ethanol was added and mixed by pipetting. The suspension was centrifuged for 2 minutes at 13,000 rpm, and the supernatant decanted and discarded. Further centrifugation for 2 minutes at 13,000 rpm was performed, and the supernatant removed by pipetting. The resultant pellet was resuspended in $30 \mu \mathrm{L}$ of $70 \%$ formic acid, followed by an equal volume of acetonitrile. The suspension was mixed by pipetting, and then centrifuged for 2 minutes at 13,000 rpm. Finally, the supernatant was collected and used for AP-MALDI analysis.

An in-house made protein standard mixture consisting of bovine cytochrome $\mathrm{C}$, bovine ubiquitin, equine myoglobin and porcine insulin was run by liquid MALDI MS prior to each experiment to ensure the instrument was satisfactorily working. The peptide standard mixture was prepared as per manufacturer's guidelines and used as a standard for both solid and liquid MALDI MS analysis.

An ethylene glycol-based LSM was used for all liquid MALDI samples. This was prepared using a $25 \mathrm{mg} / \mathrm{mL}$ - -cyano-4-hydroxycinnamic acid (CHCA) or 2,5-dihydroxybenzoic acid 
(DHB) solution in acetonitrile and water (70:30, v:v), using vortexing and sonication to dissolve the matrix chromophore crystals, followed by the addition of $70 \%$ ethylene glycol and vortexing. Liquid MALDI samples were spotted onto a stainless-steel target plate, starting with $0.5 \mu \mathrm{L}$ of $\mathrm{LSM}$ solution, then adding $0.5 \mu \mathrm{L}$ of the analyte solution (bacterial extract, protein standard or peptide standard).

For solid MALDI, a CHCA matrix solution was prepared at a concentration of $15 \mathrm{mg} / \mathrm{mL}$ using acetonitrile and water (70:30, v:v) with $0.1 \%$ trifluoroacetic acid as solvent. Similarly, a DHB matrix solution was prepared at a concentration of $25 \mathrm{mg} / \mathrm{mL}$ using the same solvent. Solid MALDI samples were spotted onto the stainless-steel target plate, starting with $1 \mu \mathrm{L}$ of analyte solution followed by $1 \mu \mathrm{L}$ of matrix solution. The solid MALDI sample was left to dry at room temperature prior to analysis.

For calibrating the time-of-flight (TOF) mass analyzer, a liquid sample droplet containing $0.5 \mu \mathrm{L}$ sodium iodide MS calibration solution $\left(2 \mu \mathrm{g} / \mathrm{mL}\right.$ in $1: 1$ isopropanol: $\left.\mathrm{H}_{2} \mathrm{O}, \mathrm{v}: \mathrm{v}\right)$ and $0.5 \mu \mathrm{L}$ of a solution of water, acetonitrile and ethylene glycol (3:7:6, v:v:v) was prepared. Calibration was performed over the $\mathrm{m} / \mathrm{z}$ range of $100-2000$.

\subsection{Liquid AP-MALDI MS Analysis}

All MS data were acquired on a Synapt G2-Si (Waters) in positive TOF mode coupled to an in-house built MALDI source. Details of the instrumental setup have been described in a previous report (20). A potential of $+3 \mathrm{kV}$ was applied between the target plate and heated transfer tube. The ion source was operated at $3.5 \mathrm{kV}$, with $180 \mathrm{~L} / \mathrm{h} \mathrm{N} \mathrm{N}_{2}$ counter-gas flow. A pulsed nitrogen laser (337nm wavelength, 3ns pulse duration) was used at a laser pulse repetition rate of $10 \mathrm{~Hz}$ at $18 \mu \mathrm{J} /$ pulse. 
Collision-induced dissociation (CID) was used for MS/MS analysis with argon as the collision gas. MS/MS data was acquired using a trap collision energy of $30 \mathrm{~V}$ (28).

\subsection{MS Data Analysis}

All data were processed using MassLynx 4.1 (Waters) software. AMX Model Builder (Waters) was used to perform PCA on the data. Deconvolution of mass spectral data was performed by UniDec software (29).

\subsection{Ethical Approval}

Ethical approval was not required for this study as the research undertaken did not involve human or animal material.

\section{Results}

\subsection{Comparison of Liquid and Solid MALDI MS Analysis of Bacterial Extracts}

A single bacterial extract was selected for MS analysis with both a solid MALDI sample and a liquid MALDI sample. Figure 1A shows the spectrum obtained for Escherichia coli following MS analysis from a solid MALDI sample. The spectrum contains an abundance of highintensity MALDI matrix-related ions, found in clusters spaced 211 Da apart, decreasing in intensity as the $\mathrm{m} / \mathrm{z}$ value increases. The mass difference of $211 \mathrm{Da}$ can be attributed to the addition of a sodiated deprotonated CHCA molecule. In general, cluster matrix ion signals for CHCA are typically formed as a result of sodium and potassium ions in varying compositions as previously noted by Smirnov et al. (30). 
MALDI MS analysis of lipids typically employs matrix chromophore compounds such as DHB $(31,32)$ or 9-aminoacridine (33-35) rather than CHCA. Thus, the bacterial extracts were also analyzed by AP-MALDI MS using DHB (Supplemental Material, Figure S2). However, no improvement was obtained in solid AP-MALDI MS, with the DHB cluster ions being the dominant ion signals, while the mass spectra obtained from DHB-based liquid MALDI samples revealed lower abundance and less variety for lipid ion signals and no ion signals for peptides or proteins when compared to the spectra obtained from CHCA-based liquid MALDI samples.

Spectra of the peptide calibration standard were also recorded from solid MALDI samples to demonstrate the efficacy of solid AP-MALDI on the MS instrument used for this study (Supplemental Material, Figure S1). All peptides that were expected to be detected within the given $\mathrm{m} / \mathrm{z}$ range of up to 2000 were recorded as singly charged ions using solid APMALDI. No multiply charged ions of these or higher molecular mass peptides were detected. However, lipid ions from the bacterial extracts were not detected by solid AP-MALDI MS.

On the other hand, when E. coli is analyzed using a liquid MALDI sample, the resultant spectrum contains a unique fingerprint of lipids in the $\mathrm{m} / \mathrm{z}$ range of $500-850$. Above $\mathrm{m} / \mathrm{z}$ 850, five peaks from multiply charged ions are present in the spectrum. Deconvolution of these peaks indicate these are the $[\mathrm{M}+8 \mathrm{H}]^{8+},[\mathrm{M}+7 \mathrm{H}]^{7+},[\mathrm{M}+6 \mathrm{H}]^{6+},[\mathrm{M}+5 \mathrm{H}]^{5+}$ and $[\mathrm{M}+4 \mathrm{H}]^{4+}$ ions from the same protein or protein fragment, with a software-calculated average mass of 7180 Da.

\subsection{Liquid AP-MALDI MS Profiling of Multiple Bacterial Strains}

A selection of 10 bacterial species were analyzed using liquid AP-MALDI, including Escherichia coli, Klebsiella pneumoniae, Campylobacter jejuni, Staphylococcus aureus, 
Staphylococcus epidermidis, Streptococcus pyogenes, Lactobacillus brevis, Enterococcus faecalis, Enterococcus hirae and Pseudomonas aeruginosa. Typical mass spectra from their analysis are shown in Figure 2. Visual inspection of these spectra already shows that each species possesses a unique lipid profile in the $\mathrm{m} / \mathrm{z}$ range of $400-1100$. However, many peaks observed in this region are common to multiple bacterial species albeit in varying relative abundances. Putative identification of the lipid ion peaks has been performed using the open access LIPID MAPS structure database (Supplemental Material, Table S2) (36).

\subsection{MS/MS Analysis of E. coli Lipid Profile Peaks}

To confirm the suspected identity of putative lipid profile peaks in the selected $\mathrm{m} / \mathrm{z}$ ranges, MS/MS analysis was performed. Figure 3 shows the MS/MS spectrum of the precursor ion at $\mathrm{m} / \mathrm{z} 726.5$, which can be attributed to the sodium adduct of a phosphatidylethanolamine (PE) lipid. Evidence for this identification can be found in the loss of $43 \mathrm{Da}$ (presumably $\mathrm{C}_{2} \mathrm{H}_{5} \mathrm{~N}$ from the head group), resulting in a peak at $\mathrm{m} / \mathrm{z} 683.5$, and the loss of $141 \mathrm{Da}$ (ethanolamine phosphate head group), resulting in a peak at $\mathrm{m} / \mathrm{z} 585.5$ - both are characteristic fragments of PEs as reported earlier(37). Other fragmentation detected in the MS/MS spectrum include the putative loss of fatty acid groups $\mathrm{C} 16: 0$ and $\mathrm{C} 17: 1$ as seen as a loss of $256 \mathrm{Da}$ and $268 \mathrm{Da}$, respectively, from the fragment ion at $\mathrm{m} / \mathrm{z} 683.44$ (see Figure 3). All of the above peaks have been previously identified by Zhang et al. (37).

\subsection{Principle Component Analysis}

Raw MS profile data files were imported to the AMX Model Builder software for PCA. For each of the ten species, nine data files acquired from three biological replicates from separate cultures analyzed in triplicates were added to the model, and the $\mathrm{m} / \mathrm{z}$ range 400 1100 was selected for multivariate statistics. Classification performance of the AMX model 
was assessed by the software's built-in 'leave $20 \%$ out' cross validation method, reporting a correct classification rate of $97.78 \%$. Figure 4 shows a plot of the PCA data for the first 3 principle components (PC1, PC2 and PC3). Data points for each species form separate clusters, allowing species discrimination. The peaks associated with the highest influence on variation in PC1 and PC2 are tabulated in the Supplemental Material (Table S3).

\section{Discussion}

Each bacterial species in this study produced a unique lipid MS profile when analyzed using liquid AP-MALDI MS. In clinical MS biotyping instruments, lipid profiles are typically not analyzed, as these instruments are optimized for the analysis of the unique peptidomic/proteomic fingerprint of microbial extracts, detecting mainly ribosomal proteins and their fragments in the $\mathrm{m} / \mathrm{z}$ range of $2,000-12,000$. The analytical sensitivity of such instruments is commonly enhanced by using axial TOF mass analyzers in the linear mode with ion deflection devices or lower detector voltages for lowering the detection of the $\mathrm{m} / \mathrm{z}$ range in which lipids are detected. Thus, lipids are excluded from the analysis in these instruments, arguably due to reasons associated with excessive ion suppression from matrix cluster formation when using solid MALDI and detector saturation in the low $\mathrm{m} / \mathrm{z}$ range as it is often the case for axial MALDI-TOF instruments operated in the linear mode.

As shown, by using liquid AP-MALDI the lower $\mathrm{m} / \mathrm{z}$ range is far less populated with highintensity matrix cluster ions as observed with solid AP-MALDI in this study (see Figure 1) and solid MALDI as reported in the literature (30). E. coli extracts analyzed by solid AP-MALDI MS only revealed matrix cluster ions, providing no diagnostic information.

The use of liquid AP-MALDI coupled to an orthogonal Q-TOF mass analyzer also overcomes some of the limitation due the potentially excessive desorption of neutral matrix 
compounds and clusters as well as late matrix ion cluster formation due to sodium- and potassium-mediated cation formation, which can pose far greater detector saturation issues in linear mode axial TOF mass analyzers. Thus, lipids and metabolites occupying the same $\mathrm{m} / \mathrm{z}$ range as matrix/cation clusters will be easier to detect with orthogonal hybrid instrumentation that can effectively decouple the source from the analyzer for the purpose of limiting the amount of matrix clusters reaching the detector.

Some hybrid MS instrumentation like the one used in this study also allow ion mobility separation, which particularly for lipids can provide another dimension of separation and will be further investigated in future studies. Ion mobility has the capability to separate ions based on their collisional cross section, which can provide the separation of isobaric species (38), as well as a substantial reduction of chemical background noise (39).

As lipids are essential components of the cell membrane, with roles in protein localization which is vital to the bacterial life cycle (40), the detection of their ion signals provides valuable diagnostic information, allowing to distinguish bacteria based on their lipid profile $(15,37)$.

A diverse set of bacteria were selected for analysis in this study to account for species diversity in demonstrating the use of liquid AP-MALDI MS in bacterial classification for a genetically varied group of organisms. Some of the selected species are of high clinical importance. Amongst these, S. aureus, K. pneumoniae and P. aeruginosa are part of a group named ESKAPE pathogens, which are organisms that have a high potential to resist actions of antibiotics (41). Closely related species were also included to test how well these can be distinguished using their lipid profiles. Thus, S. aureus and S. epidermidis, as well as E. hirae and $E$. faecalis were included as these bacteria respectively belong to the same genus and 
possess relatively similar lipid profiles. Also, a high degree of similarity can be seen between certain species, such as $E$. coli and $K$. pneumoniae, with an almost identical lipid profile. These were distinguishable by the intensities of their lipid ion peaks. As both species are member of the same family, Enterobacteriaeceae, the close resemblance of their lipid profiles can be expected. Similar lipid MS profiles have also been observed in other studies for Enterobacteriaeceae, with the distribution of PEs significantly varying based on their genus (42). However, here it has been shown that it is possible to achieve species-level discrimination.

A marked difference in the profile pattern can be seen for lipid profiles obtained for grampositive and gram-negative bacteria. Previous MS studies by Zhang et al. have shown that spectra of gram-negative bacteria contain fatty acids, lyso-phospholipids, phosphatidylethanolamines (PE) and phosphatidylglycerols (PG) in abundance, while spectra of gram-positive bacteria contain lipopeptides, and with limited abundance lysophospholipids (37). This difference can also be seen in the distribution of the lipid profiles between the gram-positive and gram-negative species used in this study. All gram-positive bacteria investigated (Figure 2E-J) exhibit a lipid profile in the $\mathrm{m} / \mathrm{z}$ range of $900-1000$, whereas gram-negative species show the highest intensity distribution of lipid ion signals in the $\mathrm{m} / \mathrm{z}$ range of $650-800$. This is consistent with MS studies that demonstrate that gramnegative bacteria have a high ion signal abundance of PEs and PGs within this range (43). Gram-positive bacteria exhibit a lipid profile in a higher $\mathrm{m} / \mathrm{z}$ range, due to the presence of different lipid classes, such as lysophospholipids present in S. aureus (44) and cardiolipins found in Lactobacillus species (45). 
It is not only the lipid profile that can be potentially exploited using liquid AP-MALDI MS. In Figure $1 \mathrm{~B}$, the higher $\mathrm{m} / \mathrm{z}$ range shows that multiply charged ions can be obtained from bacterial samples. The estimated molecular weight of the underlying molecular species is $7180 \mathrm{Da}$, possibly a ribosomal protein or protein fragment as typically detected in commercial MALDI MS biotyping instruments (46). The detection of proteins using MALDI and axial-TOF instrumentation is the current standard for mass spectrometry in clinical microbiology, and therefore the acquisition of lipid ion signals alongside peptide and protein ion signals contributes valuable diagnostic information.

In this study, species-level discrimination has been achieved for all investigated bacteria using lipid profiles. However, there might be species that cannot be distinguished based on their lipid MS profile alone. In these cases, the ability of liquid AP-MALDI MS to detect lipid profiles in combination with some multiply charged peptide and protein ions can further improve bacterial biotyping, potentially allowing the discrimination between closely related species and strain-level identification, which could allow tracking of strain evolution and acquisition of resistance profiles.

Abundant ion signals for lipids and proteins in hybrid MS instruments also allow superior MS/MS analysis compared to current commercial MS biotyping instrumentation, thus providing structural information for these macromolecules. As clinical biotyping of microorganisms is performed on axial MALDI-TOF mass spectrometers, MS/MS analysis and therefore bacterial identification is principally inferior. The use of the AP-MALDI source and Q-TOF mass analyzer in this study allows the acquisition of high-quality MS/MS data, providing further highly specific sequence information for peptides and proteins from the same analysis without additional sample preparation. Protocols for optimised 
lipid/peptide/protein extraction and the combined lipid profile and sequence analysis are currently under development and will be reported elsewhere.

In summary, this study has investigated the application of liquid AP-MALDI MS on a hybrid Q-TOF MS instrument for the analysis of bacterial extracts. In a clinical laboratory, bacterial identification by mass spectrometry is typically performed based on a unique proteomic fingerprint. The data presented here shows that there is the potential to perform specieslevel identification based on the unique lipid MS profile of the bacteria, which is typically not obtained from commercial MALDI-TOF instruments approved for clinical analysis. It is important to note that environmental factors can influence the lipid composition in bacteria. Thus, further studies with respect to the influence of the growth medium, temperature and incubation time are needed.

The ability of liquid AP-MALDI to generate multiply charged peptide/protein ions through its application on hybrid high-performing MS/MS instrumentation will undoubtedly add analytical power to the detection of lipid MS profiles. Ultimately, this option should provide bacterial identification of higher confidence compared to current instruments approved for clinical use.

Data supporting the results reported in this paper are openly available from the University of Reading Research Data Archive at http://dx.doi.org/10.17864/1947.221. 
Author contributions: All the authors have accepted responsibility for the entire content of this submitted manuscript and approved submission.

Research funding: This work was supported by EPSRC through grant EP/R513301/1.

Employment or leadership: None declared.

Honorarium: None declared.

Competing interests: The funding organization played no role in the study design; in the collection, analysis, and interpretation of data; in writing of the report; or in the decision to submit the report for publication.

Acknowledgements: Access to the AMX Model Builder software was kindly provided by Waters Corporation. 


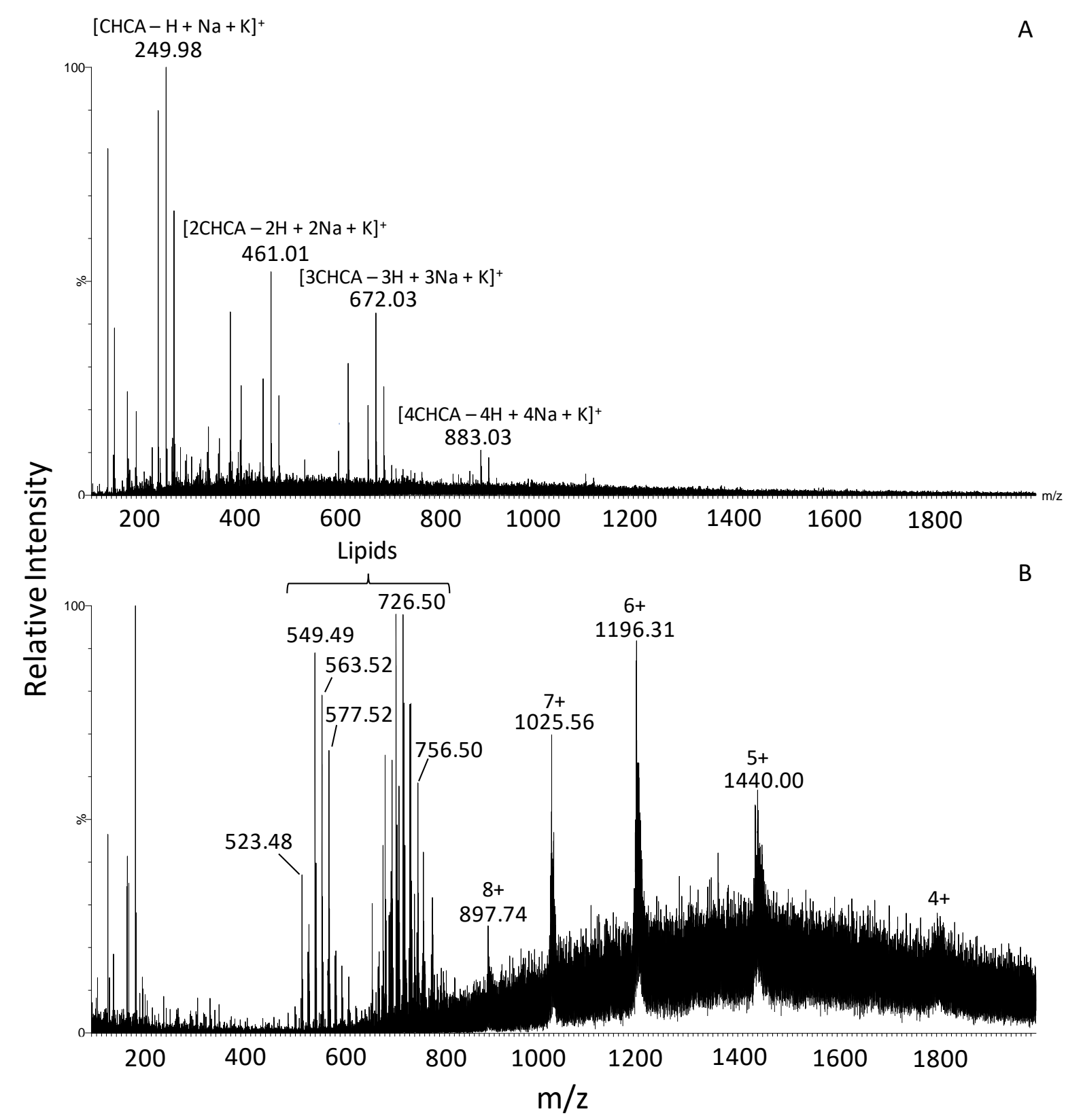

Figure 1 - AP-MALDI mass spectra of E. coli obtained from a solid (A) and liquid (B) MALDI sample, where CHCA represents the matrix chromophore compound. The most abundant isotopologues are labelled. 


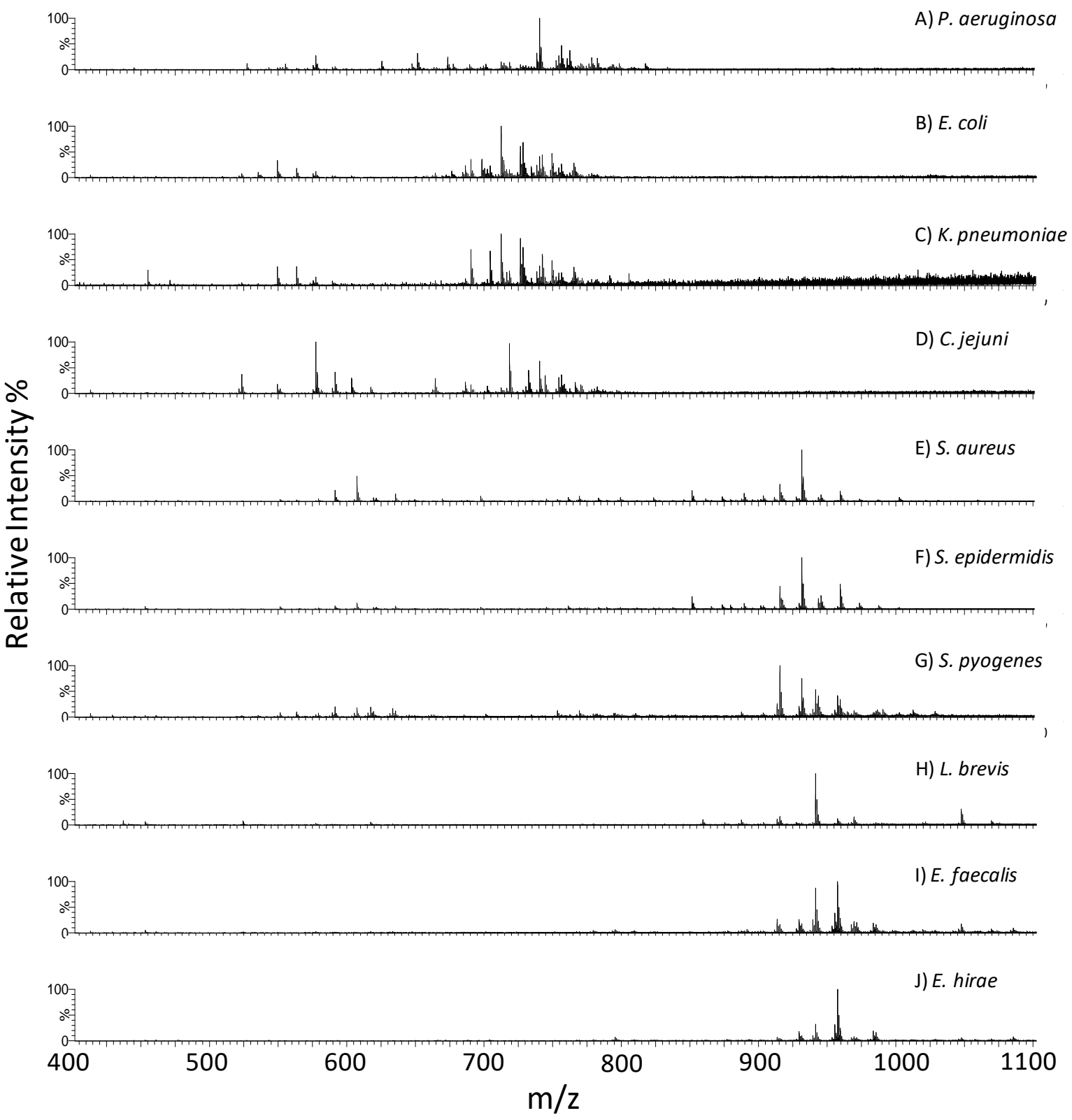

Figure 2 - Liquid AP-MALDI mass spectra of the lipid profiles of several bacterial species in positive ion mode. 
$[\mathrm{PE}(33: 1)+\mathrm{Na}]^{+}$



[PE(33:1)-Head Group $]^{+}$ 563.49

[PE(33:1)-Head Group+Na $]^{+}$

/ 585.47

$\left[\mathrm{PE}(33: 1)-\mathrm{C}_{2} \mathrm{H}_{5} \mathrm{~N}+\mathrm{Na}\right]^{+}$

683.44

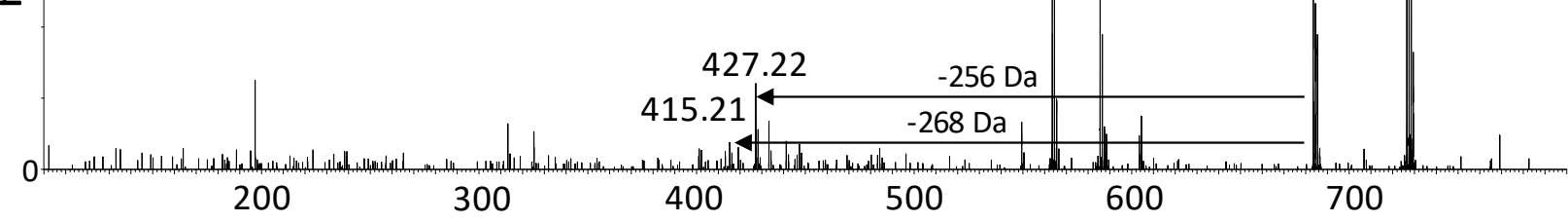

$\mathrm{m} / \mathrm{z}$

Figure 3 - Liquid AP-MALDI MS/MS spectrum of the precursor ion at $\mathrm{m} / \mathrm{z} 726.5$, detected in the lipid MS profile of $E$. coli. 


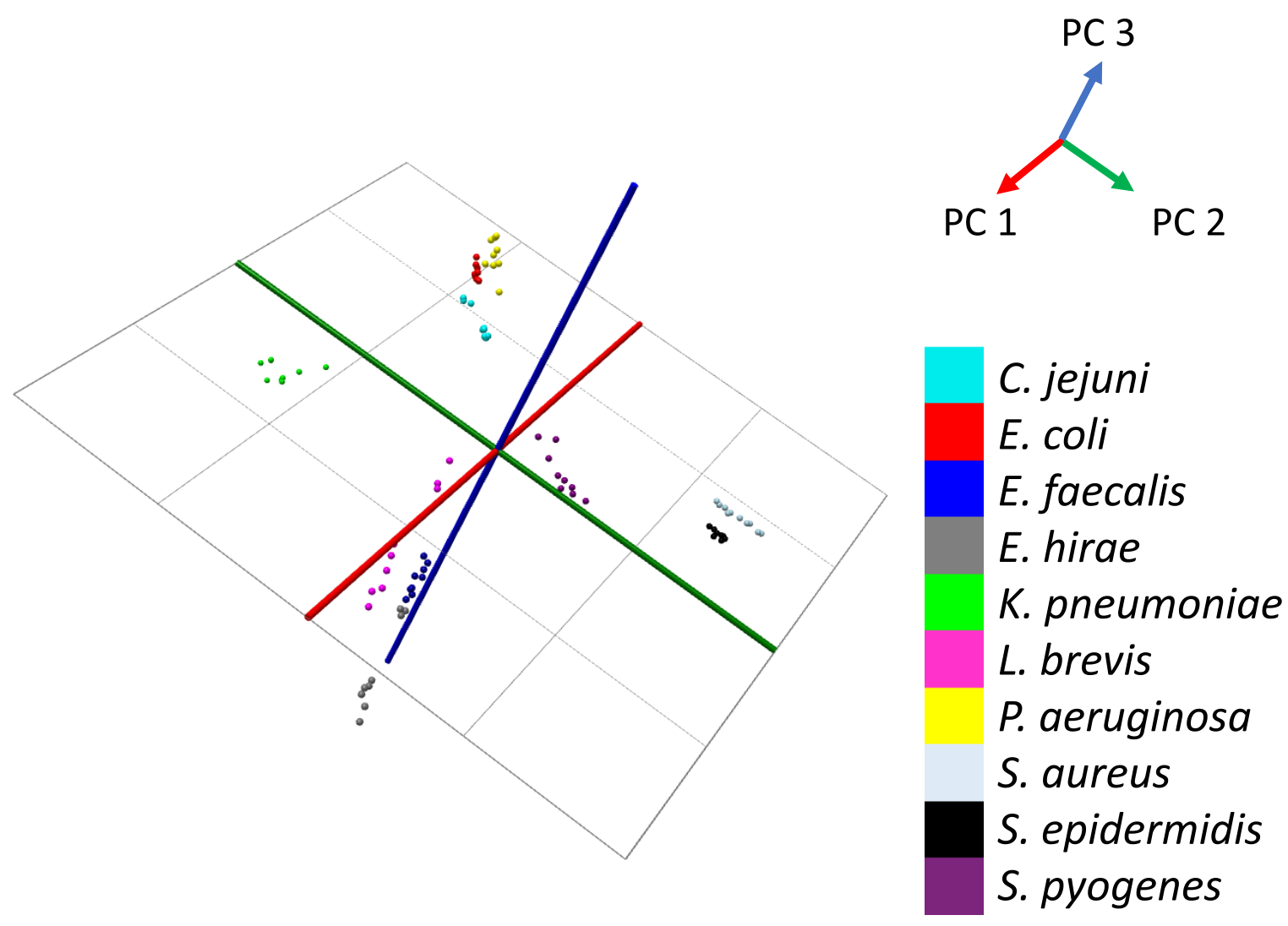

Figure 4 - Plot of the PCA data points for the first three principle components (PC1, PC2 and PC3) using MS profile data from 10 bacterial species. 


\section{References}

1. Carbonnelle E, Mesquita C, Bille E, Day N, Dauphin B, Beretti J-L, et al. MALDI-TOF mass spectrometry tools for bacterial identification in clinical microbiology laboratory. Clinical Biochemistry. 2011;44(1):104-9.

2. Croxatto A, Prod'hom G, Greub G. Applications of MALDI-TOF mass spectrometry in clinical diagnostic microbiology. FEMS Microbiology Reviews. 2012;36(2):380-407.

3. Perez KK, Olsen RJ, Musick WL, Cernoch PL, Davis JR, Land GA, et al. Integrating Rapid Pathogen Identification and Antimicrobial Stewardship Significantly Decreases Hospital Costs. Archives of Pathology and Laboratory Medicine. 2013;137(9):1247-54.

4. $\quad$ Patel TS, Kaakeh R, Nagel JL, Newton DW, Stevenson JG. Cost Analysis of Implementing Matrix-Assisted Laser Desorption Ionization-Time of Flight Mass Spectrometry Plus Real-Time Antimicrobial Stewardship Intervention for Bloodstream Infections. Journal of Clinical Microbiology. 2017;55(1):60-7.

5. Faron ML, Buchan BW, Hyke J, Madisen N, Lillie JL, Granato PA, et al. Multicenter Evaluation of the Bruker MALDI Biotyper CA System for the Identification of Clinical Aerobic Gram-Negative Bacterial Isolates. PLOS ONE. 2015;10(11):e0141350.

6. Seng P, Drancourt M, Gouriet F, Scola BL, Fournier PE, Rolain JM, et al. Ongoing revolution in bacteriology: Routine identification of bacteria by matrix-assisted laser desorption ionization timeof-flight mass spectrometry. Clinical Infectious Diseases. 2009;49(4):543-51.

7. Han X, Gross RW. Global analyses of cellular lipidomes directly from crude extracts of biological samples by ESI mass spectrometry: A bridge to lipidomics. Journal of Lipid Research. 2003;44(6):1071-9.

8. Dworzanski JP, Berwald L, Meuzelaar HLC. Pyrolytic Methylation-Gas Chromatography of Whole Bacterial Cells for Rapid Profiling of Cellular Fatty Acids. Applied and Environmental Microbiology. 1990;56(6):1717-24.

9. Basile F, Voorhees KJ, Hadfield TL. Microorganism gram-type differentiation based on pyrolysis-mass spectrometry of bacterial Fatty Acid methyl ester extracts. Applied and Environmental Microbiology. 1995;61(4):1534-9.

10. Dworzanski JP, Tripathi A, Snyder AP, Maswdeh WM, Wick CH. Novel biomarkers for Gramtype differentiation of bacteria by pyrolysis-gas chromatography-mass spectrometry. Journal of Analytical and Applied Pyrolysis. 2005;73(1):29-38.

11. Fang J, Barcelona MJ. Structural determination and quantitative analysis of bacterial phospholipids using liquid chromatography/electrospray ionization/mass spectrometry. Journal of Microbiological Methods. 1998;33(1):23-35.

12. Smith PBW. Characterization of Bacterial Phospholipids by Electrospray Ionization Tandem Mass Spectrometry. Analytical Chemistry. 1995;67(11):1824-30.

13. Gidden J, Denson J, Liyanage R, Ivey DM, Lay JO. Lipid Compositions in Escherichia coli and Bacillus subtilis During Growth as Determined by MALDI-TOF and TOF/TOF Mass Spectrometry. International Journal of Mass Spectrometry. 2009;283(1-3):178-84.

14. Hamid AM, Jarmusch AK, Pirro V, Pincus DH, Clay BG, Gervasi G, et al. Rapid Discrimination of Bacteria by Paper Spray Mass Spectrometry. Analytical Chemistry. 2014;86(15):7500-7.

15. Heller DN, Cotter RJ, Fenselau C, Uy OM. Profiling of bacteria by fast atom bombardment mass spectrometry. Analytical Chemistry. 1987;59(23):2806-9.

16. Strittmatter N, Rebec M, Jones EA, Golf O, Abdolrasouli A, Balog J, et al. Characterization and Identification of Clinically Relevant Microorganisms Using Rapid Evaporative Ionization Mass Spectrometry. Analytical Chemistry. 2014;86(13):6555-62.

17. Shu X, Li Y, Liang M, Yang B, Liu C, Wang Y, et al. Rapid lipid profiling of bacteria by online MALDI-TOF mass spectrometry. International Journal of Mass Spectrometry. 2012;321-322:71-6. 
18. Cramer R, Pirkl A, Hillenkamp F, Dreisewerd K. Liquid AP-UV-MALDI Enables Stable Ion Yields of Multiply Charged Peptide and Protein Ions for Sensitive Analysis by Mass Spectrometry.

Angewandte Chemie International Edition. 2013;52(8):2364-7.

19. Towers MW, Mckendrick JE, Cramer R. Introduction of 4-Chloro- $\alpha$-cyanocinnamic Acid Liquid Matrices for High Sensitivity UV-MALDI MS. Journal of Proteome Research. 2010;9(4):1931-40.

20. Ryumin P, Brown J, Morris M, Cramer R. Investigation and optimization of parameters affecting the multiply charged ion yield in AP-MALDI MS. Methods. 2016;104:11-20.

21. Ryumin P, Cramer R. The composition of liquid atmospheric pressure matrix-assisted laser desorption/ionization matrices and its effect on ionization in mass spectrometry. Analytica Chimica Acta. 2018;1013:43-53.

22. Trimpin S, Wang B, Inutan ED, Li J, Lietz CB, Harron A, et al. A Mechanism for lonization of Nonvolatile Compounds in Mass Spectrometry: Considerations from MALDI and Inlet lonization. Journal of The American Society for Mass Spectrometry. 2012;23(10):1644-60.

23. Hale OJ, Cramer R. Collision-induced dissociation of doubly-charged barium-cationized lipids generated from liquid samples by atmospheric pressure matrix-assisted laser desorption/ionization provides structurally diagnostic product ions. Analytical and Bioanalytical Chemistry. 2018;410(5):1435-44.

24. Hale OJ, Morris M, Jones B, Reynolds CK, Cramer R. Liquid Atmospheric Pressure MatrixAssisted Laser Desorption/Ionization Mass Spectrometry Adds Enhanced Functionalities to MALDI MS Profiling for Disease Diagnostics. ACS Omega. 2019;4(7):12759-65.

25. Hale OJ, Ryumin P, Brown JM, Morris M, Cramer R. Production and analysis of multiply charged negative ions by liquid atmospheric pressure matrix-assisted laser desorption/ionization mass spectrometry. Rapid Communications in Mass Spectrometry. 2018;1(8).

26. Bruker Daltonik GmbH. Instructions for Use - MALDI Biotarget 48. Available from: https://www.bruker.com/fileadmin/user upload/8-PDFDocs/Separations MassSpectrometry/InstructionForUse/IFU 268711267615226413 MALDI Biot arget 48 Rev1.pdf

27. England PH. Culture Collections [National Collection of Type Cultures]. Available from: https://www.phe-culturecollections.org.uk/collections/nctc.aspx.

28. Cramer R, Corless $\mathrm{S}$. The nature of collision-induced dissociation processes of doubly protonated peptides: comparative study for the future use of matrix-assisted laser desorption/ionization on a hybrid quadrupole time-of-flight mass spectrometer in proteomics. Rapid Communications in Mass Spectrometry. 2001;15(22):2058-66.

29. Marty MT, Baldwin AJ, Marklund EG, Hochberg GKA, Benesch JLP, Robinson CV. Bayesian Deconvolution of Mass and Ion Mobility Spectra: From Binary Interactions to Polydisperse Ensembles. Analytical Chemistry. 2015;87(8):4370-6.

30. Smirnov IP, Zhu X, Taylor T, Huang Y, Ross P, Papayanopoulos IA, et al. Suppression of $\alpha$ Cyano-4-hydroxycinnamic Acid Matrix Clusters and Reduction of Chemical Noise in MALDI-TOF Mass Spectrometry. Analytical Chemistry. 2004;76(10):2958-65.

31. Vieler A, Wilhelm C, Goss R, Süß R, Schiller J. The lipid composition of the unicellular green alga Chlamydomonas reinhardtii and the diatom Cyclotella meneghiniana investigated by MALDITOF MS and TLC. Chemistry and Physics of Lipids. 2007;150(2):143-55.

32. Jackson SN, Wang H-YJ, Woods AS. Direct Profiling of Lipid Distribution in Brain Tissue Using MALDI-TOFMS. Analytical Chemistry. 2005;77(14):4523-7.

33. Cerruti CD, Benabdellah F, Laprévote O, Touboul D, Brunelle A. MALDI Imaging and Structural Analysis of Rat Brain Lipid Negative Ions with 9-Aminoacridine Matrix. Analytical Chemistry. 2012;84(5):2164-71.

34. Angelini R, Vitale R, Patil VA, Cocco T, Ludwig B, Greenberg ML, et al. Lipidomics of intact mitochondria by MALDI-TOF/MS. Journal of Lipid Research. 2012;53(7):1417-25. 
35. Angelini R, Babudri F, Lobasso S, Corcelli A. MALDI-TOF/MS analysis of archaebacterial lipids in lyophilized membranes dry-mixed with 9-aminoacridine. Journal of Lipid Research.

2010;51(9):2818-25.

36. Structure Database (LMSD) LIPID MAPS (R) Lipidomics Gateway. Available from: https://www.lipidmaps.org/data/structure/LMSDSearch.php?Mode=SetupTextOntologySearch.

37. Zhang JI, Talaty N, Costa AB, Xia Y, Tao WA, Bell R, et al. Rapid direct lipid profiling of bacteria using desorption electrospray ionization mass spectrometry. International Journal of Mass Spectrometry. 2011;301(1):37-44.

38. Woods AS, Ugarov M, Egan T, Koomen J, Gillig KJ, Fuhrer K, et al. Lipid/Peptide/Nucleotide Separation with MALDI-Ion Mobility-TOF MS. Analytical Chemistry. 2004;76(8):2187-95.

39. Venne K, Bonneil E, Eng K, Thibault P. Improvement in Peptide Detection for Proteomics Analyses Using NanoLC-MS and High-Field Asymmetry Waveform lon Mobility Mass Spectrometry. Analytical Chemistry. 2005;77(7):2176-86.

40. Barák I, Muchová K. The role of lipid domains in bacterial cell processes. International Journal Molecular Science. 2013;14(2):4050-65.

41. Pendleton JN, Gorman SP, Gilmore BF. Clinical relevance of the ESKAPE pathogens. Expert Review of Anti-infective Therapy. 2013;11(3):297-308.

42. Ishida Y, Madonna AJ, Rees JC, Meetani MA, Voorhees KJ. Rapid analysis of intact phospholipids from whole bacterial cells by matrix-assisted laser desorption/ionization mass spectrometry combined with on-probe sample pretreatment. Rapid Communications in Mass Spectrometry. 2002;16(19):1877-82.

43. Ishida Y, Kitagawa K, Nakayama A, Ohtani H. Complementary analysis of lipids in whole bacteria cells by thermally assisted hydrolysis and methylation-GC and MALDI-MS combined with onprobe sample pretreatment. Journal of Analytical and Applied Pyrolysis. 2006;77(2):116-20.

44. Peschel A, Jack RW, Otto M, Collins LV, Staubitz P, Nicholson G, et al. Staphylococcus aureus resistance to human defensins and evasion of neutrophil killing via the novel virulence factor MprF is based on modification of membrane lipids with I-lysine. Journal of Experimental Medicine.

2001;193(9):1067-76.

45. Calvano CD, Zambonin CG, Palmisano F. Lipid fingerprinting of Gram-positive lactobacilli by intact cells - matrix-assisted laser desorption/ionization mass spectrometry using a proton sponge based matrix. Rapid Communications in Mass Spectrometry. 2011;25(12):1757-64.

46. Freiwald A, Sauer S. Phylogenetic classification and identification of bacteria by mass spectrometry. Nature Protocols. 2009;4(5):732. 